\title{
TRAJECTORY PATTERN MINING
}

Fosca Giannotti, Micro Nanni, Dino Pedreschi, Fabio Pinelli 


\section{Introduction}

$\square$ Nowadays data on the spatial and temporal location is objects is available.

$\square$ Gps, GSM towers, etc

$\square$ What can we do with this data? 


\section{Introduction}

$\square$ Nowadays data on the spatial and temporal location is objects is available.

$\square$ Gps, GSM towers, etc

$\square$ What can we do with this data?

$\square$ Data mine it for patterns!! (of course) 


\section{Trajectory Pattern Mining}

A way to represent Spatial patterns.

Bistro Square $\stackrel{10 \mathrm{~min}}{\longrightarrow}$ Appleton Tower $\stackrel{2 \mathrm{hr} 30 \mathrm{~min}}{\longrightarrow}$ Potterrow

Chambers Str $\stackrel{15 \mathrm{~min}}{\longrightarrow}$ The Mound $\stackrel{5 \mathrm{~min}}{\longrightarrow}$ Waverly Station 


\section{Possible uses}

$\square$ Prediction of movement

$\square$ Aggregate movement behaviour

$\square$ Region of interests discovery

$\square$ Discovery of traffic flow \& blockages 


\section{Structure of presentation}

$\square$ Mechanics

$\square$ Data structure

$\square$ Regions of Interest (ROI)

$\square$ T-Patterns using these ROI

$\square$ Results 


\section{Data structure}

$\square$ Raw Input

$\square$ List of (Lat, Long, timestamp)

$\square$ Multiple Moving Objects

$\square$ Convert
$\square($ Lat, Long, $t) \stackrel{\text { covert }}{\longrightarrow}(x, y, t)$
$(x, y) \in R^{2}$

\section{T-Patterns}

$\square=s_{0} \stackrel{\alpha_{1}}{\rightarrow} s_{1} \stackrel{\alpha_{2}}{\rightarrow} \cdots \stackrel{\alpha_{N}}{\rightarrow} s_{N}$ 


\section{Example: Raw input}

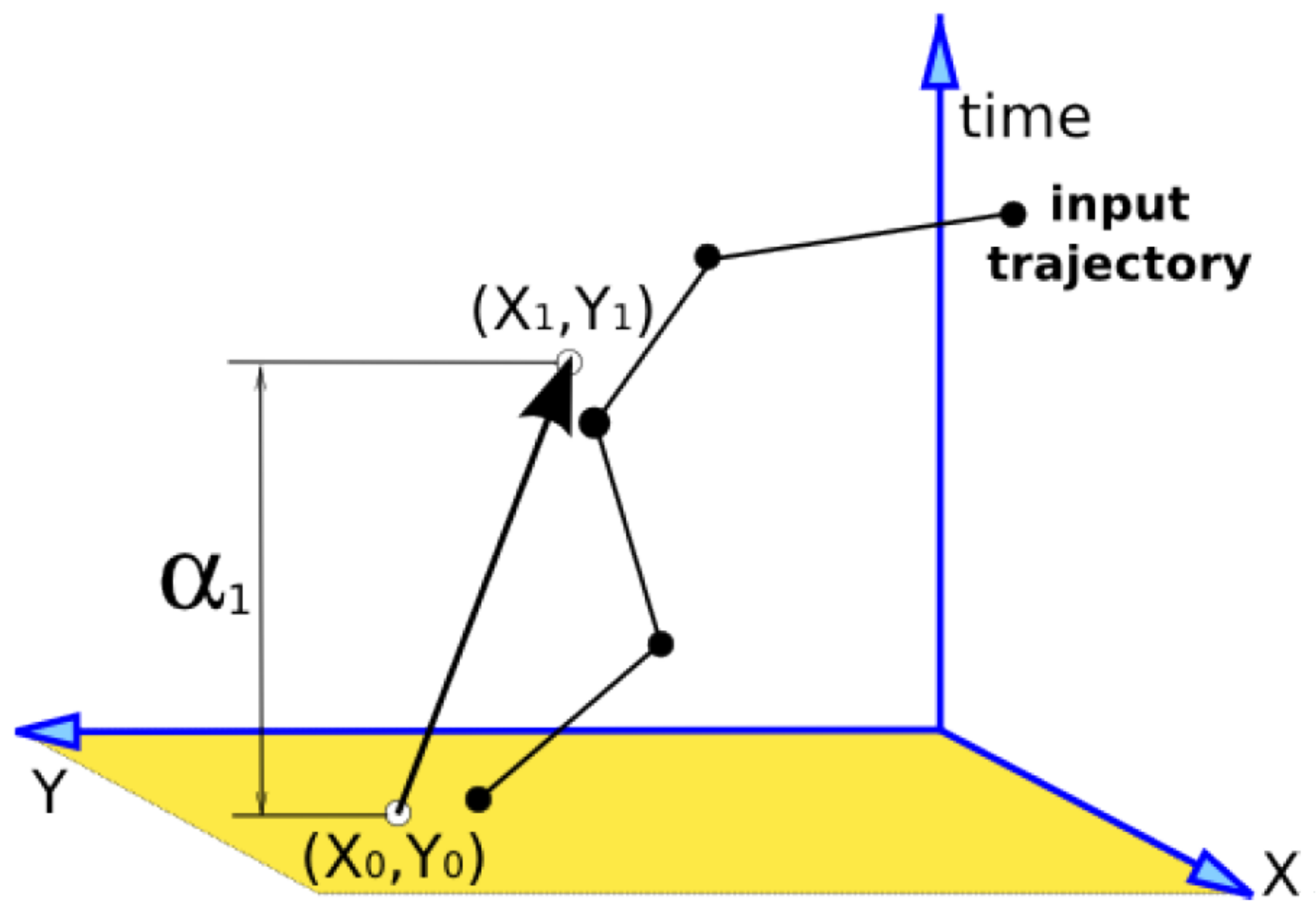

Trajectory Pattern Mining: Fosca Giannotti, Micro Nanni, Dino Pedreschi, Fabio Pinelli 


\section{Example: T-Patterns}

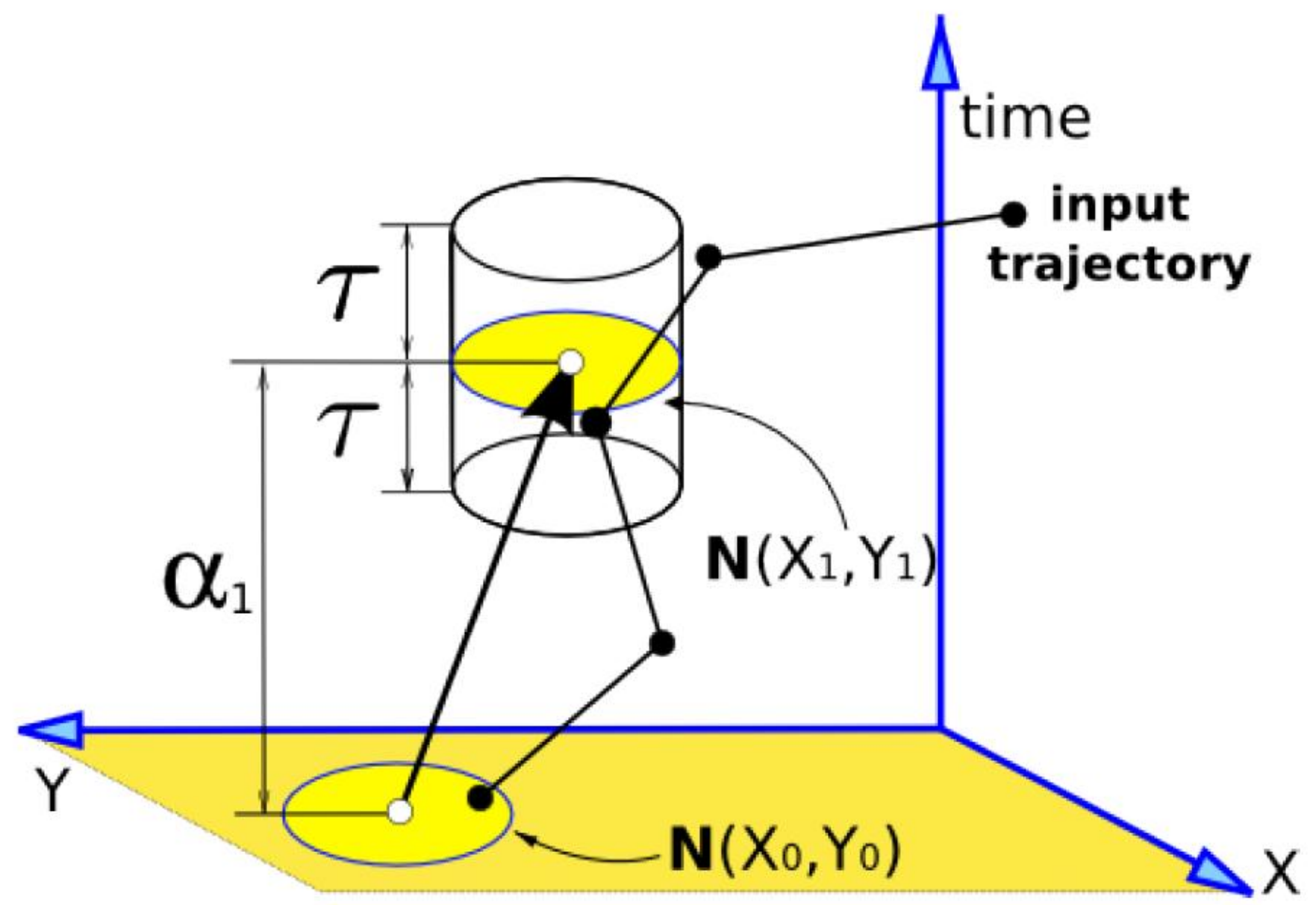

Trajectory Pattern Mining: Fosca Giannotti, Micro Nanni, Dino Pedreschi, Fabio Pinelli 


\section{Example: T-Patterns}

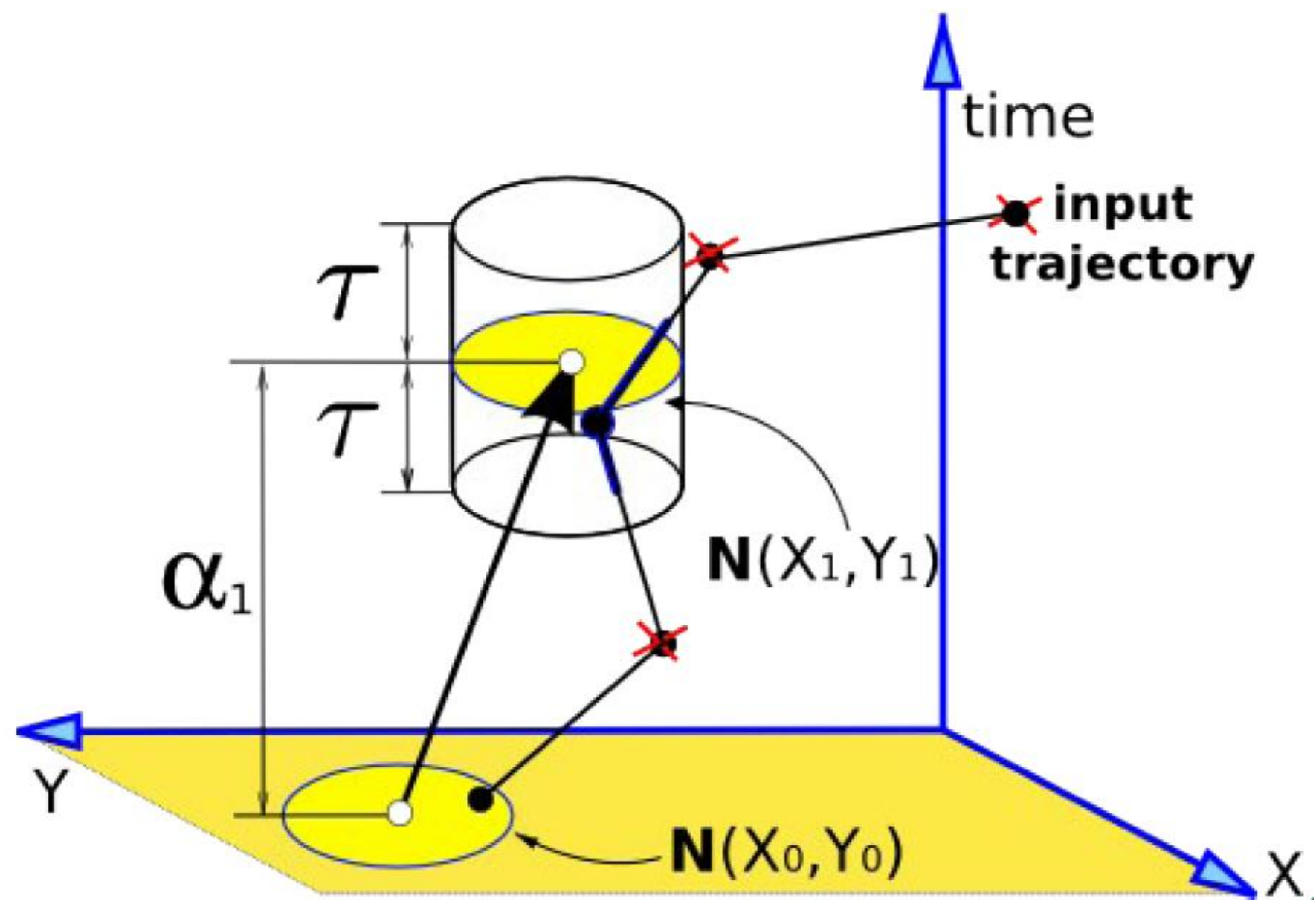

Trajectory Pattern Mining: Fosca Giannotti, Micro Nanni, Dino Pedreschi, 


\section{Support Threshold}

$\square$ Support: Percentage of trajectories which are contained within a pattern.

Example: Support threshold of 0.2.

$\square$ A T-Pattern is kept only if $20 \%$ of the trajectories support it. 


\section{Curse of Dimensionality}

$\square$ Data in 3 dimensional space $(x, y$, time).

$\square$ With Pattern length 3:

$R^{(3 \text { points } * 2 D)+(2 \text { transition } * 1 D)}$ combinations

$\square$ As pattern length increases to $n$, search space Search Space $=R^{(n * 2)+((n-1) * 1)}=R^{3 n-1}$

$\square$ Therefore intractable.

$\square$ Reduce dimensions through Regions of Interest. 


\section{Regions of Interest}

$\square$ Static pre-processed ROI

$\square$ ROI discovery

$\square$ List of candidate places

$\square$ Dynamic ROI

Popular points detection

ROI construction 


\section{Popular Points detection}

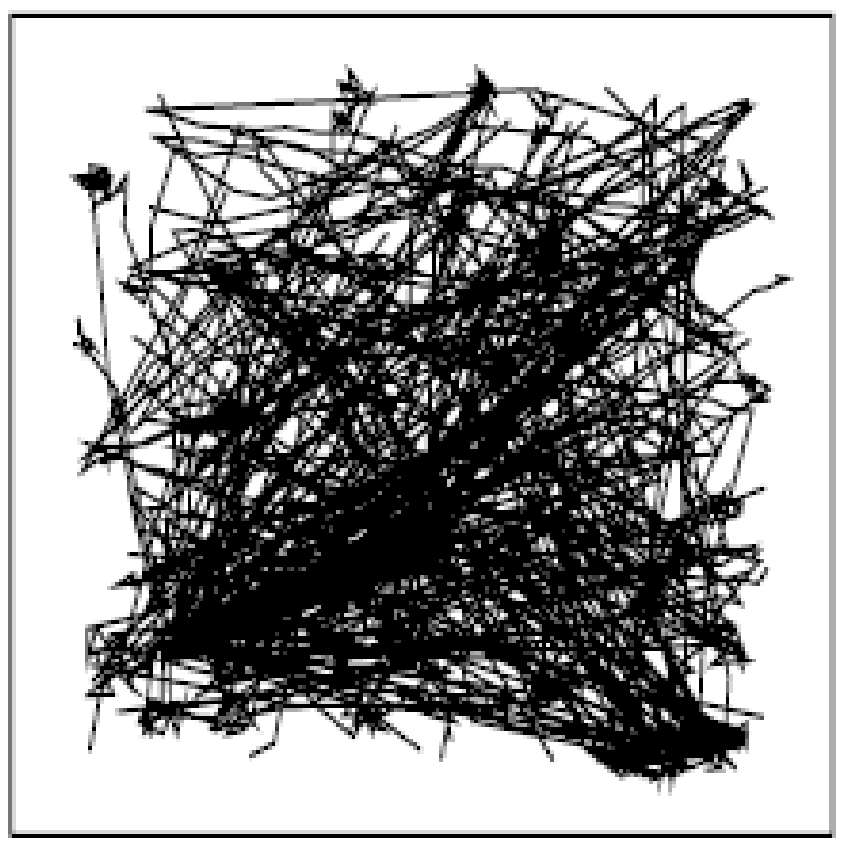

(a) input trajectories

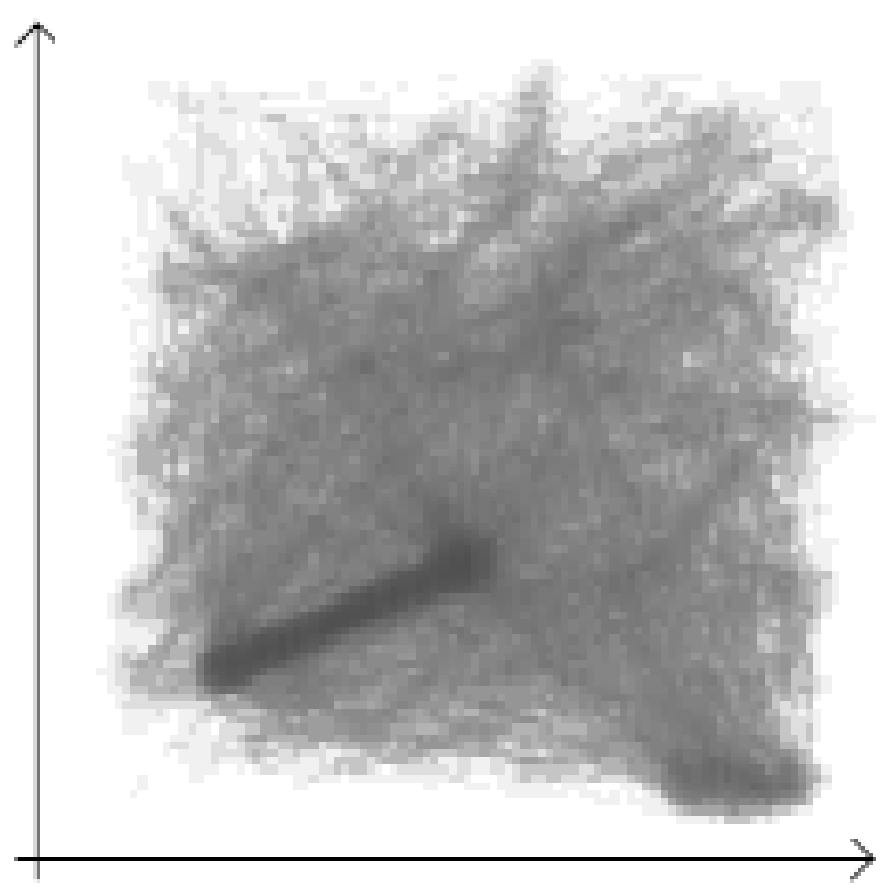

(b) density distribution

Trajectory Pattern Mining: Fosca Giannotti, Micro Nanni, Dino Pedreschi, Fabio Pinelli 


\section{ROI construction}

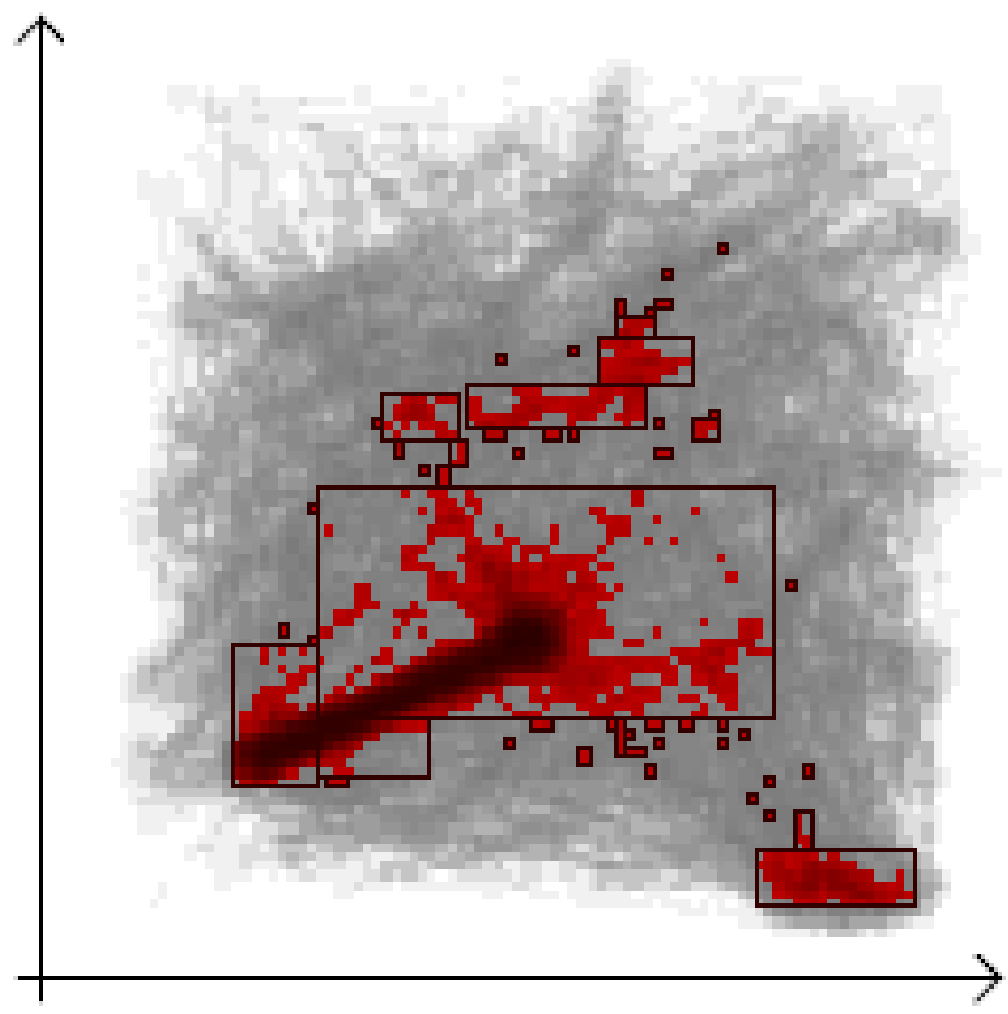

(c) dense cells and extracted RoI

Trajectory Pattern Mining: Fosca Giannotti, Micro Nanni, Dino Pedreschi,

Fabio Pinelli 


\section{T-Pattern Mining (1)}

$\square$ Two Approaches

$\square$ Static

Dynamic

$\square$ Input: Trajectories and threshold parameters

$\square$ Neighbourhood and time threshold

$\square$ Support threshold

$\square$ Output: T-Patterns 


\section{T-Pattern Mining (2)}

$\square$ Step-wise Heuristic

$\square$ Any frequent T-Pattern of length $n+1$ is the extension of some frequent T-Pattern of length $n$.

$$
\begin{gathered}
\left(x_{0}, y_{0}\right) \stackrel{\Delta t_{1}}{\longrightarrow} \cdots \stackrel{\Delta t_{n+1}}{\longrightarrow}\left(x_{n+1}, y_{n+1}\right) \preccurlyeq_{N, \tau} T \\
\left(x_{0}, y_{0}\right) \stackrel{\Delta t_{1}}{\longrightarrow} \cdots \stackrel{\Delta t_{n}}{\longrightarrow}\left(x_{n}, y_{n}\right) \preccurlyeq_{N, \tau} T
\end{gathered}
$$




\section{Static discovery of T-Patterns}

$\square$ Compute ROls over the whole trajectories.

$\square$ Covert $(x, y, t)$ trajectories to $(R O I, t)$

$\square$ Find sequences of ROls which satisfy support condition. 


\section{Dynamic Discovery of T-Patterns (1)}

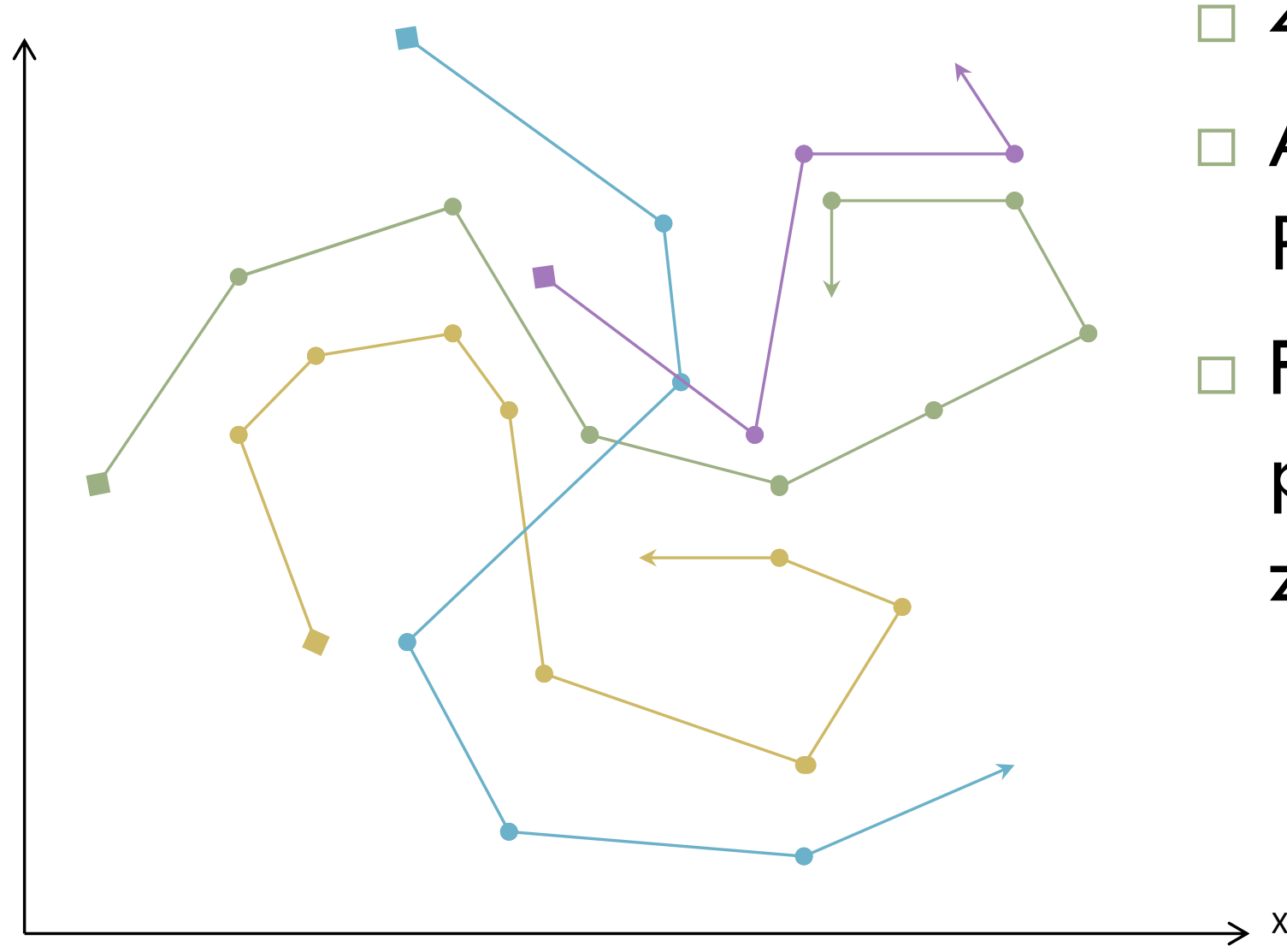

4 trajectories.

Aim is to find T-

Patterns.

First iteration prefix length is zero. 


\section{Dynamic Discovery of T-Patterns (2)}

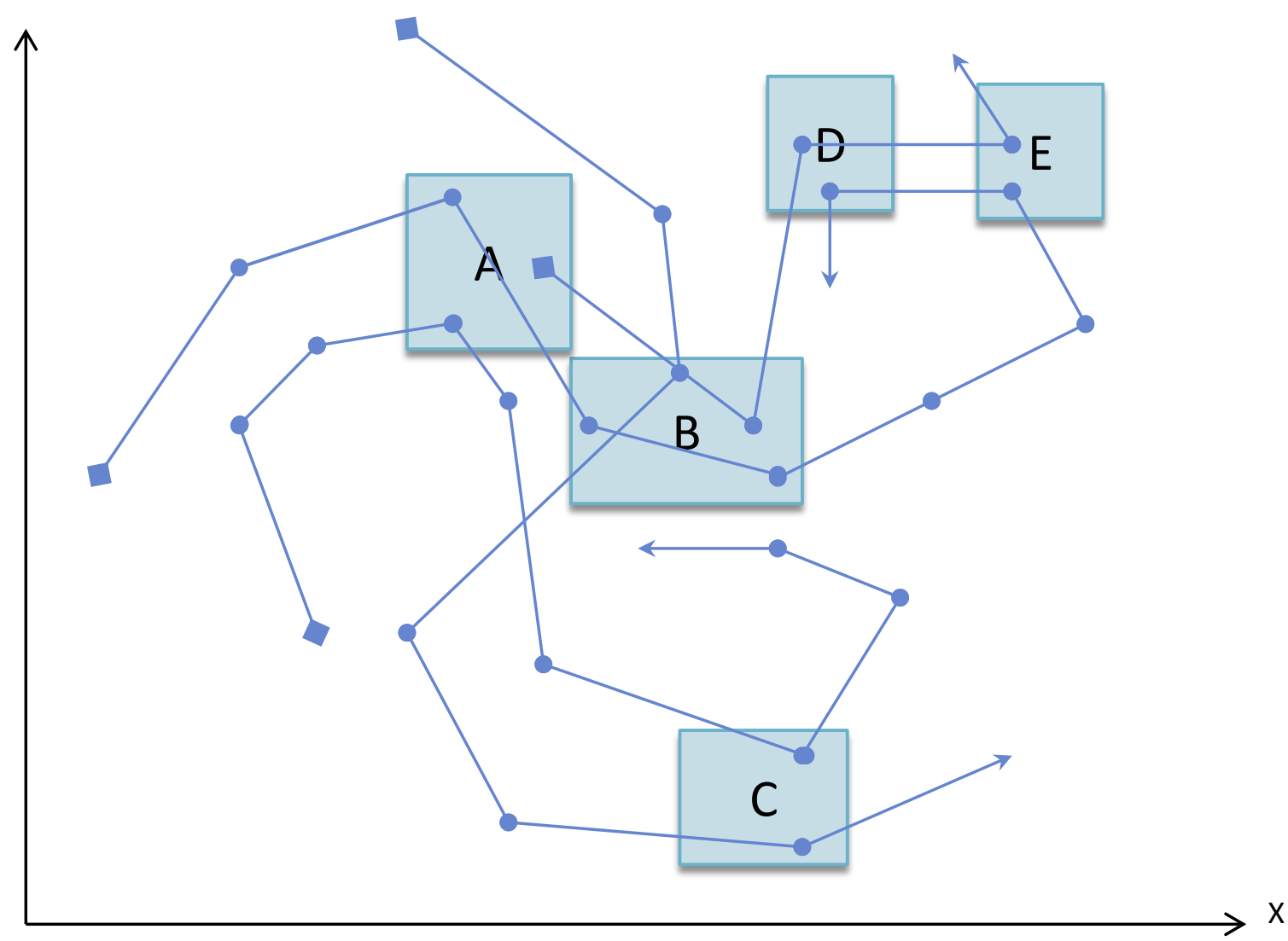

$\square$ Detect Regions of Interest

$\square$ e.g. support(r) $\geq 0.5$

$\square$ For each region find possible projections to other regions 


\section{Dynamic Discovery of T-Patterns (3)}

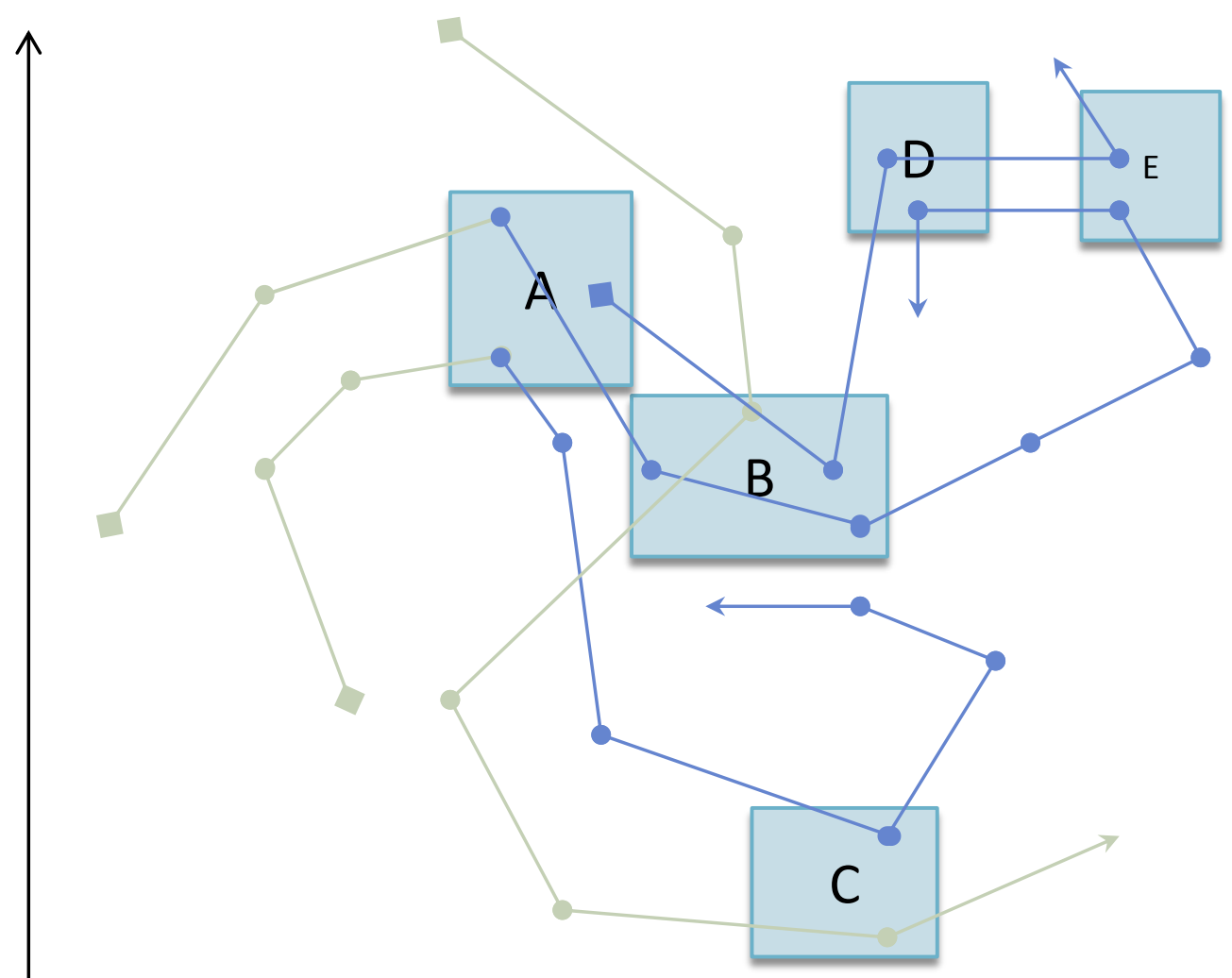

$\square$ With respect to region $A$.

$\square$ Projections:

$$
\begin{aligned}
& A \rightarrow B \\
& A \rightarrow C
\end{aligned}
$$

$\square$ Add new configuration

$\left(T^{\prime},<A>\right)$

Trajectory Pattern Mining: Fosca Giannotti, Micro Nanni, Dino Pedreschi, 


\section{Dynamic Discovery of T-Patterns (4)}

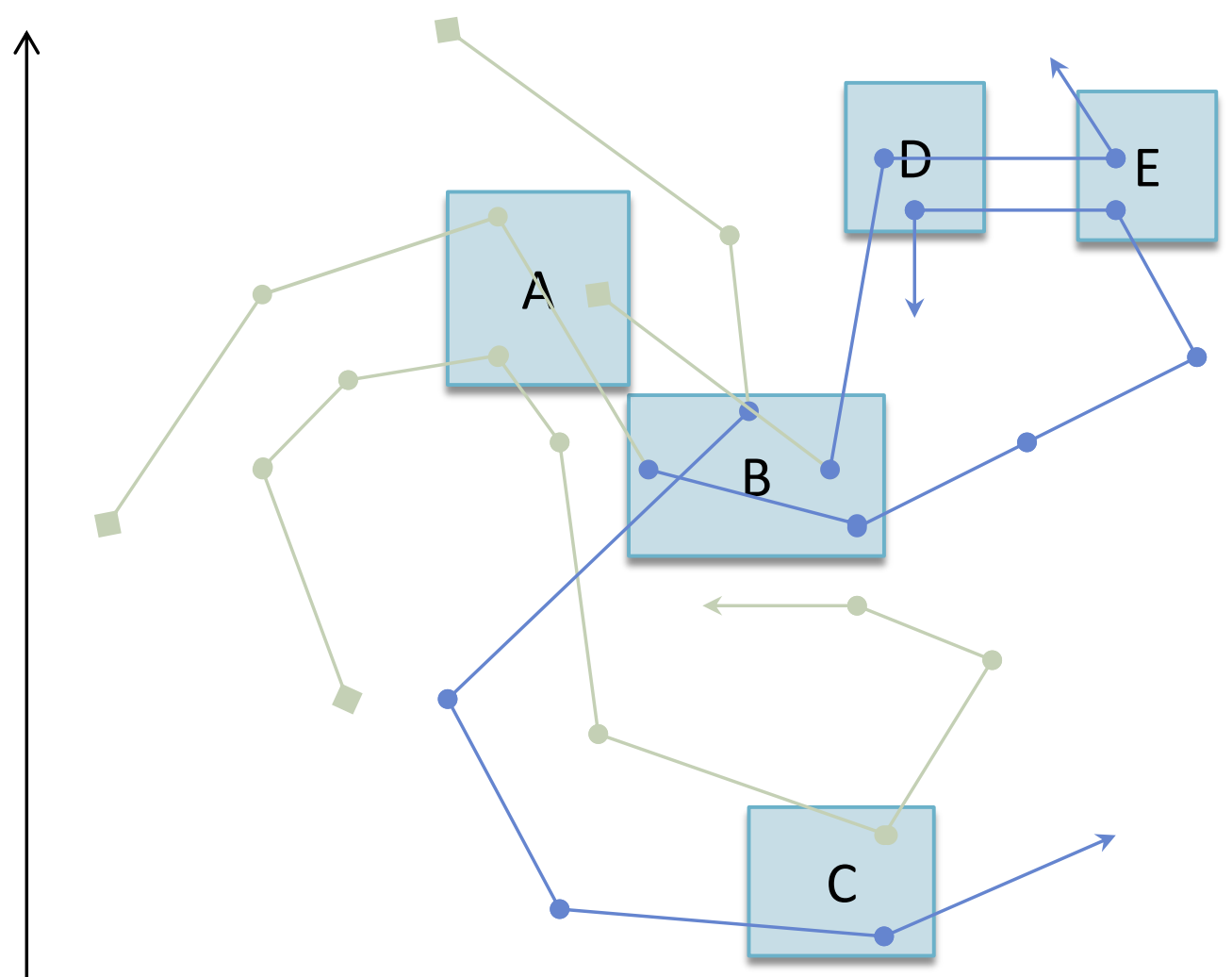

$\square$ With respect to region $B$.

$\square$ Projections:

$$
\begin{aligned}
& \mathrm{B} \rightarrow \mathrm{C} \\
& \mathrm{B} \rightarrow \mathrm{D} \\
& \mathrm{B} \rightarrow \mathrm{E}
\end{aligned}
$$

$\square$ Add new configuration

$$
\left(T^{\prime},<B>\right)
$$




\section{Dynamic Discovery of T-Patterns (5)}

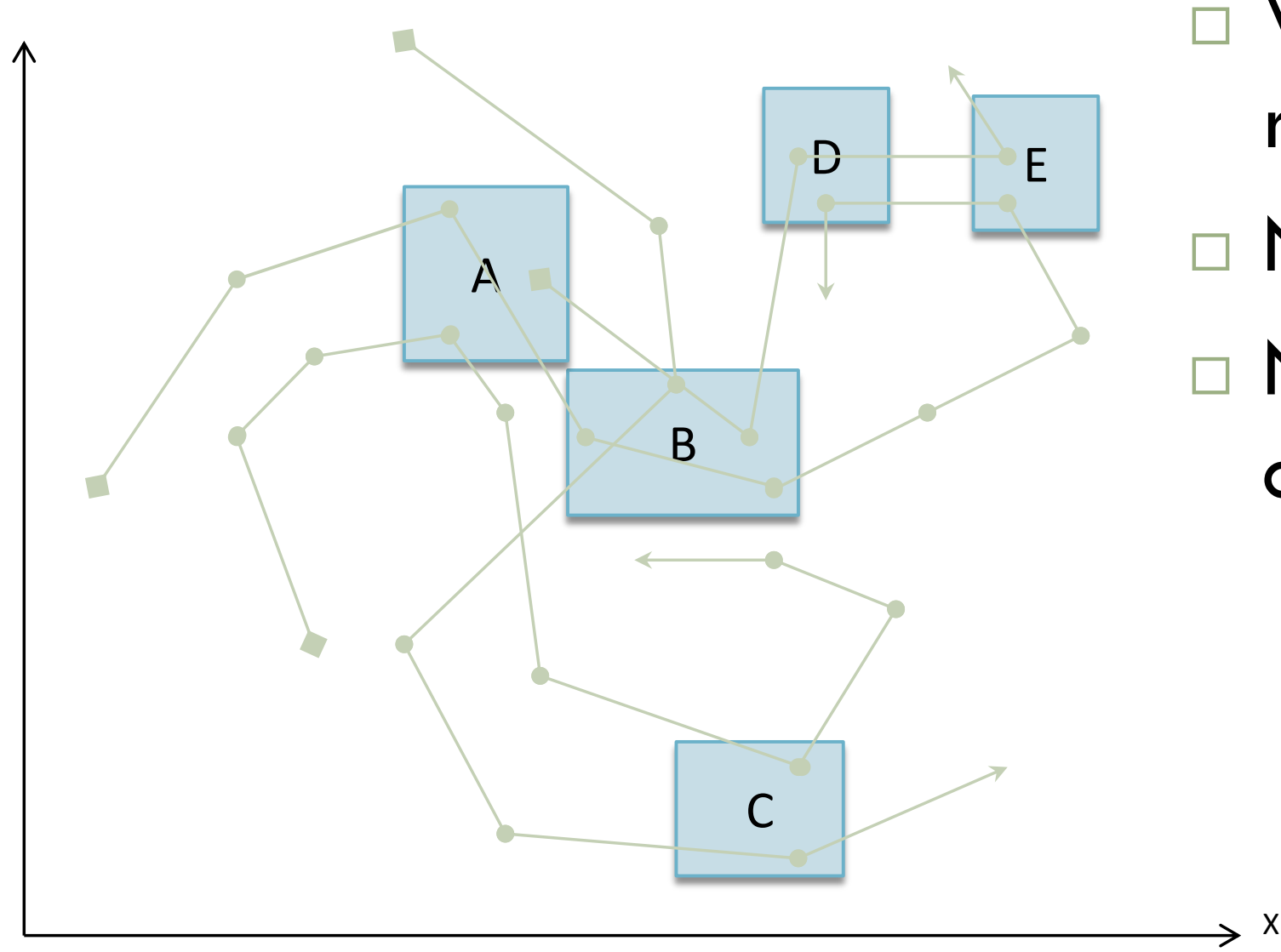

With respect to region $\mathrm{C}$.

No Projections

No new configuration

Trajectory Pattern Mining: Fosca Giannotti, Micro Nanni, Dino Pedreschi, 


\section{Dynamic Discovery of T-Patterns (6)}

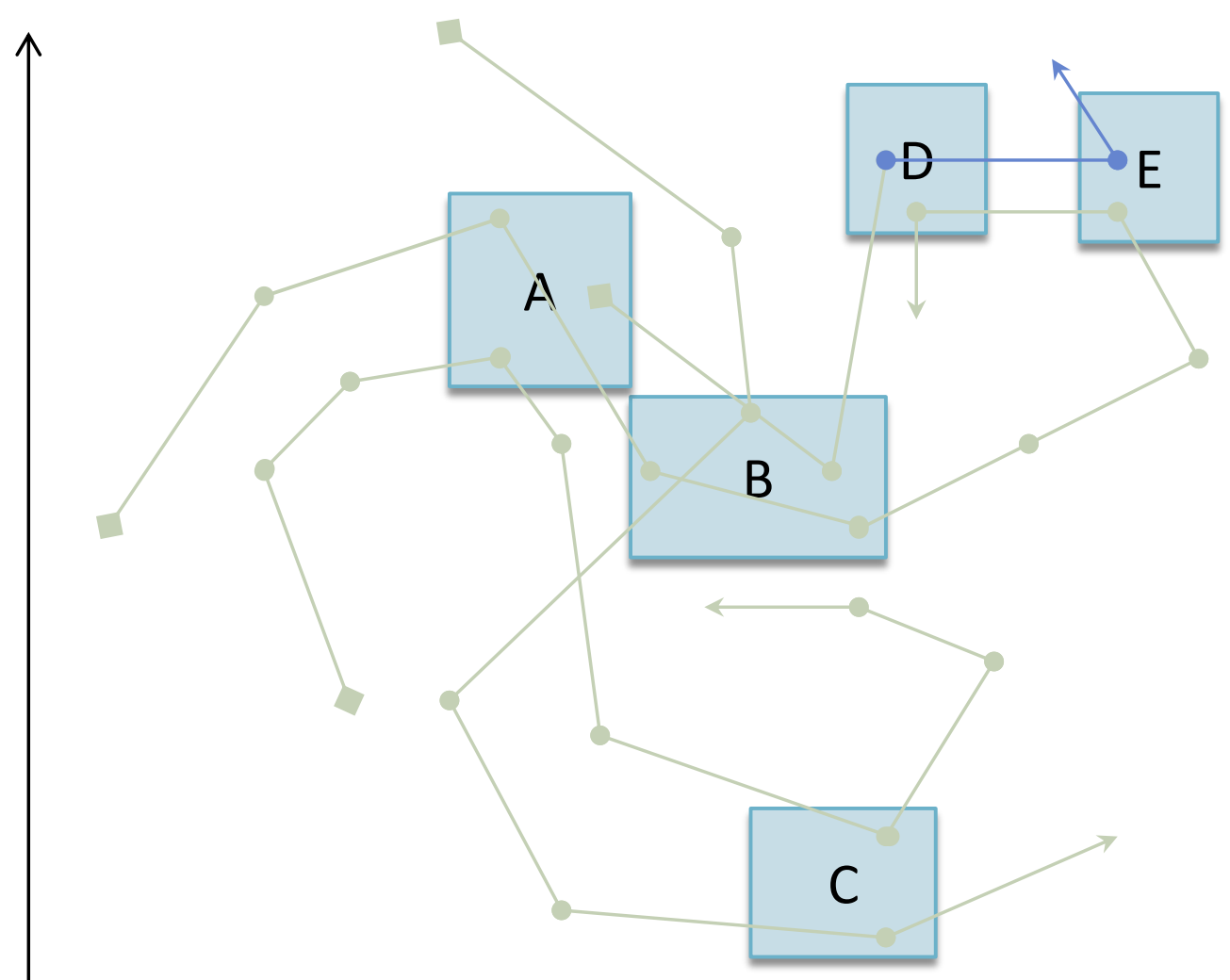

$\square$ With respect to region $D$.

$\square$ Projection

$\mathrm{D} \rightarrow \mathrm{E}$

Add new configuration

$\left(T^{\prime},<D>\right)$

Trajectory Pattern Mining: Fosca Giannotti, Micro Nanni, Dino Pedreschi, 


\section{Dynamic Discovery of T-Patterns (7)}

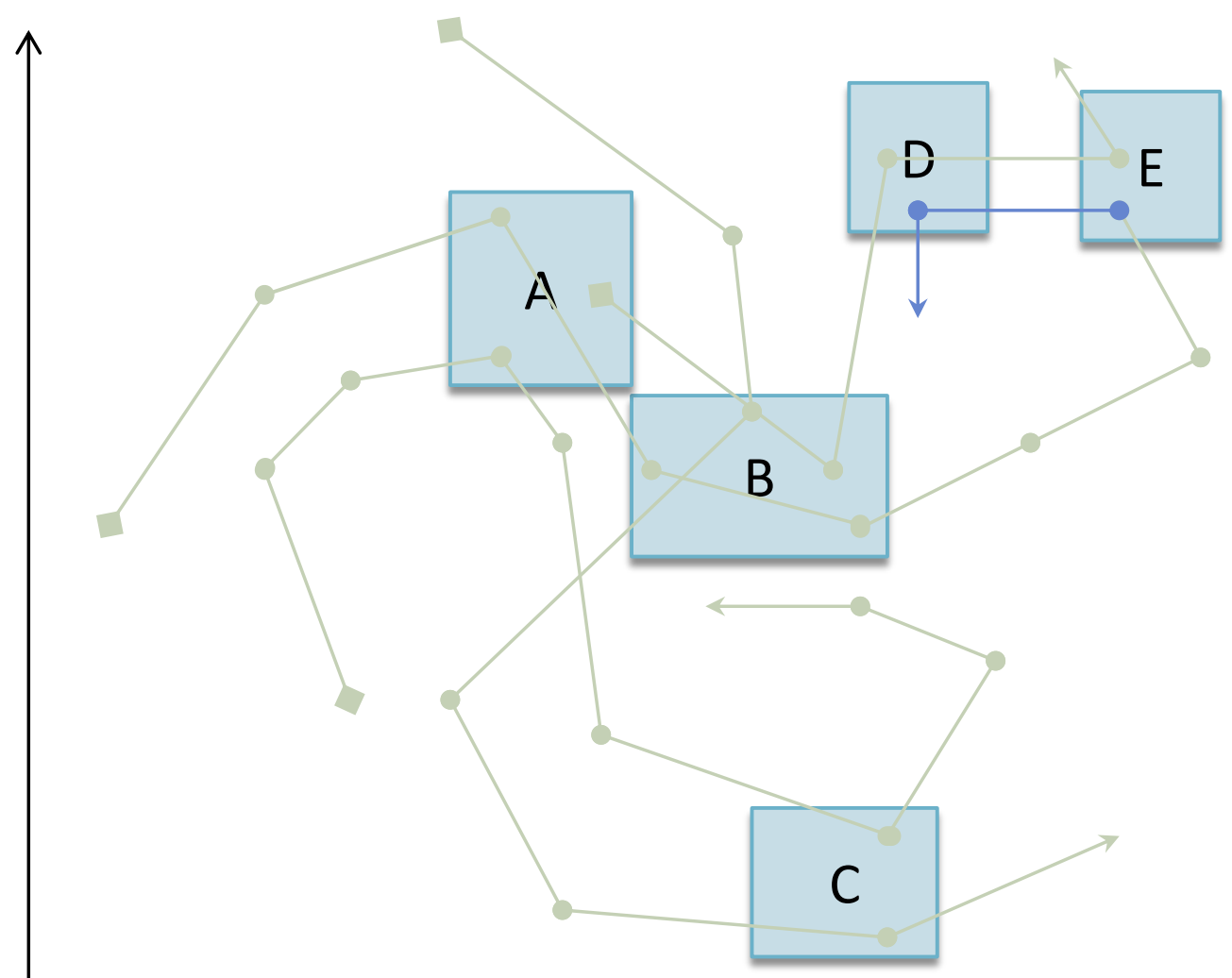

$\square$ With respect to region $\mathrm{E}$.

$\square$ Projection

$$
E \rightarrow D
$$

Add new configuration

$\left(T^{\prime},<E>\right)$

Trajectory Pattern Mining: Fosca Giannotti, Micro Nanni, Dino Pedreschi, Fabio Pinelli 


\section{Dynamic Discovery of T-Patterns (8)}

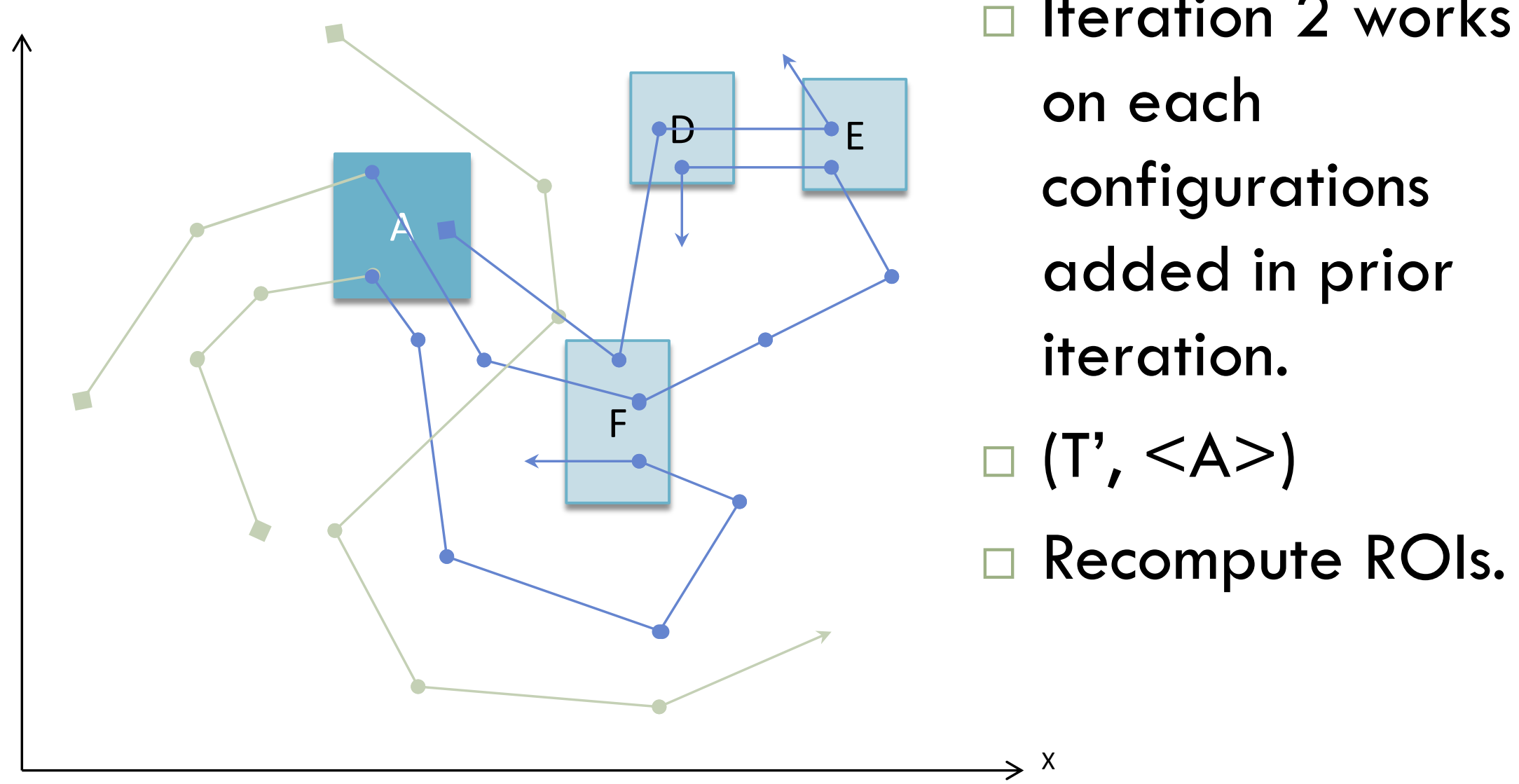

Trajectory Pattern Mining: Fosca Giannotti, Micro Nanni, Dino Pedreschi, 


\section{Dynamic Discovery of T-Patterns (9)}

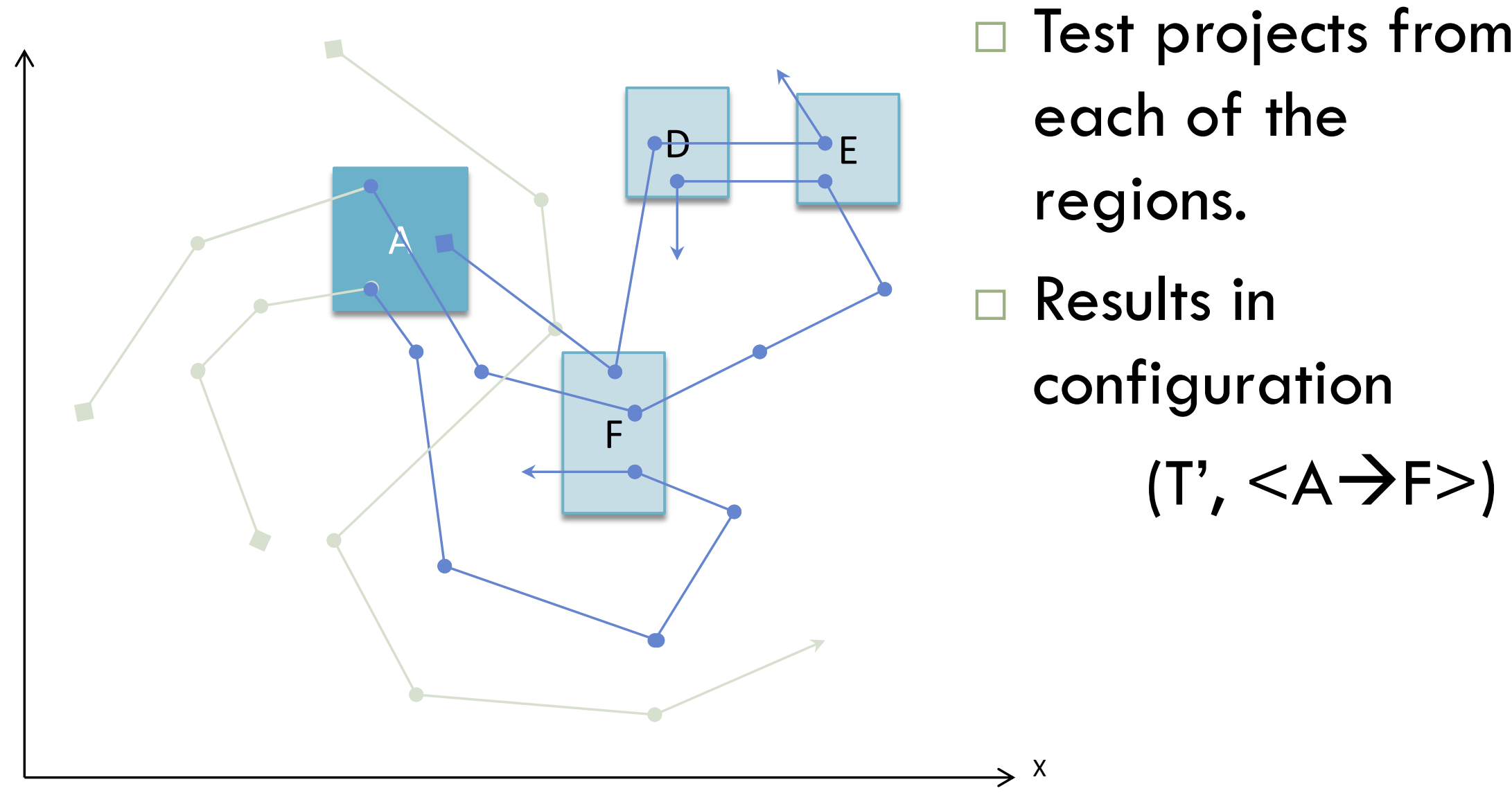

Trajectory Pattern Mining: Fosca Giannotti, Micro Nanni, Dino Pedreschi, Fabio Pinelli 


\section{Dynamic Discovery of T-Patterns (1 1 )}

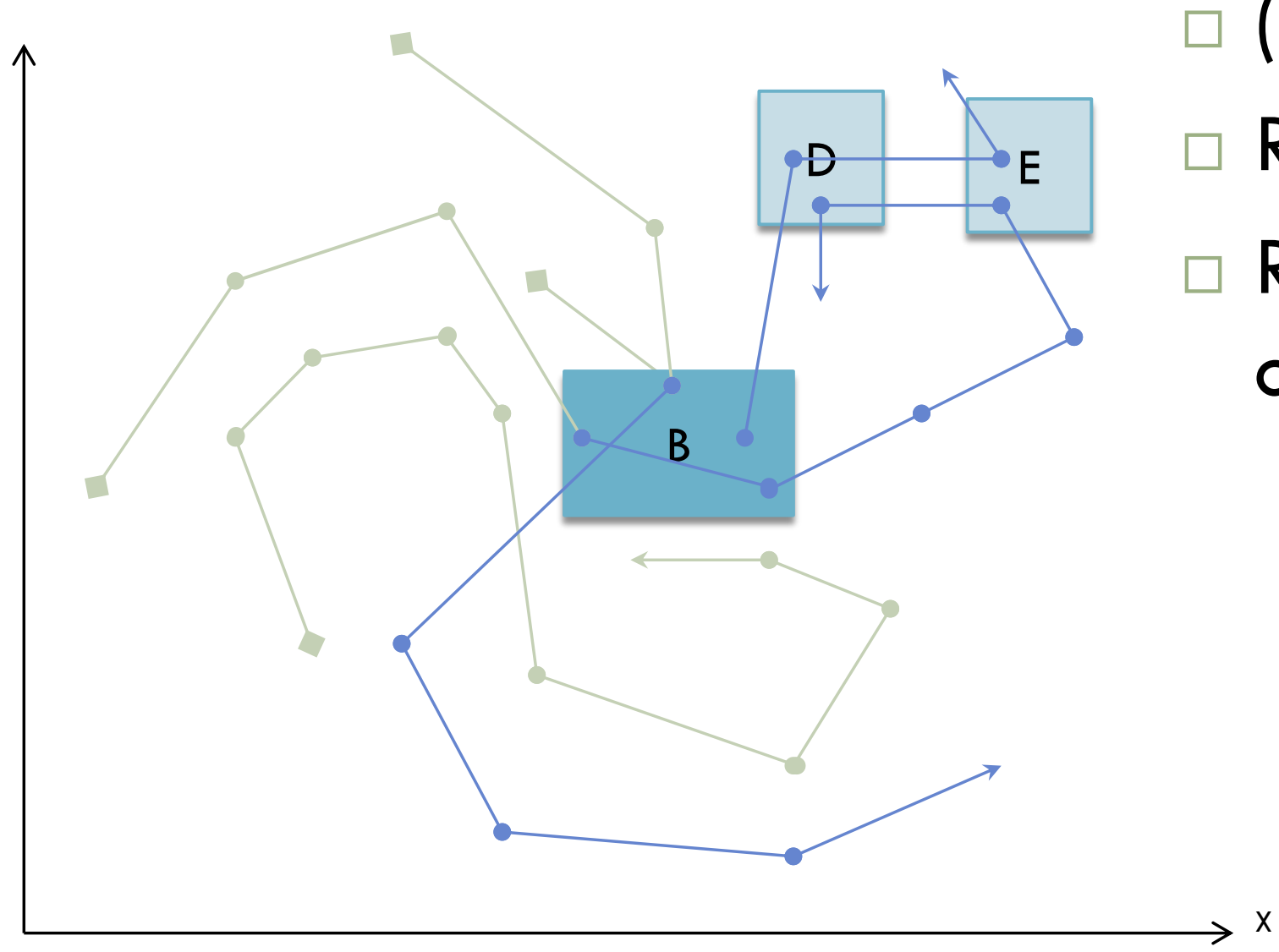

$\left(T^{\prime},<B>\right)$

Recompute ROls.

Results in configuration

$$
\begin{aligned}
& \left(T^{\prime},<B \rightarrow D>\right) \\
& \left(T^{\prime \prime},<B \rightarrow E>\right)
\end{aligned}
$$

Trajectory Pattern Mining: Fosca Giannotti, Micro Nanni, Dino Pedreschi, 


\section{Dynamic Discovery of T-Patterns (12)}

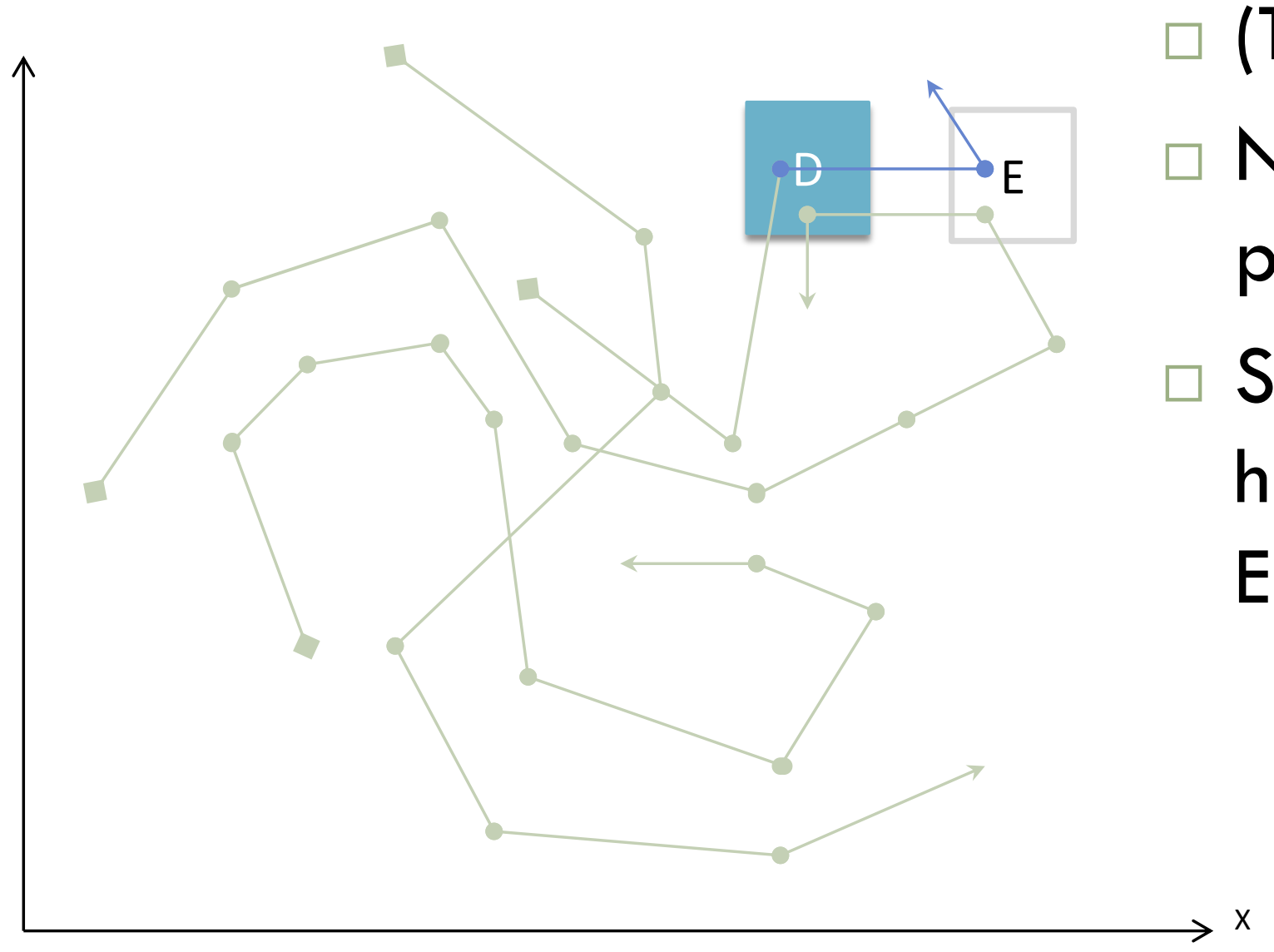

$\left(T^{\prime},<D>\right)$

No possible projections.

Same thing happens for region

E.

$x$

Trajectory Pattern Mining: Fosca Giannotti, Micro Nanni, Dino Pedreschi, 


\section{Dynamic Discovery of T-Patterns (1 1)}

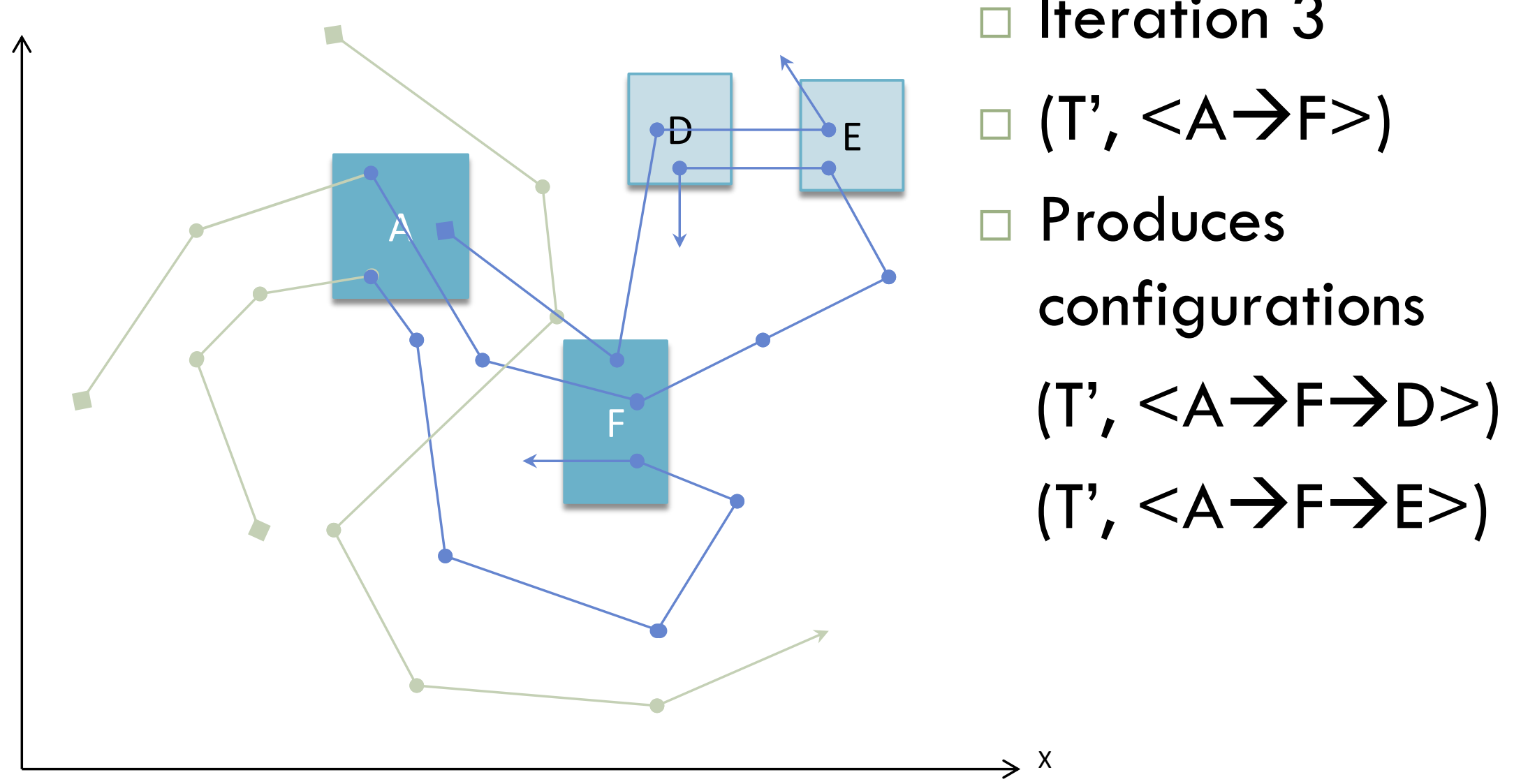

Trajectory Pattern Mining: Fosca Giannotti, Micro Nanni, Dino Pedreschi, Fabio Pinelli 


\section{Dynamic Discovery of T-Patterns (1 1 )}

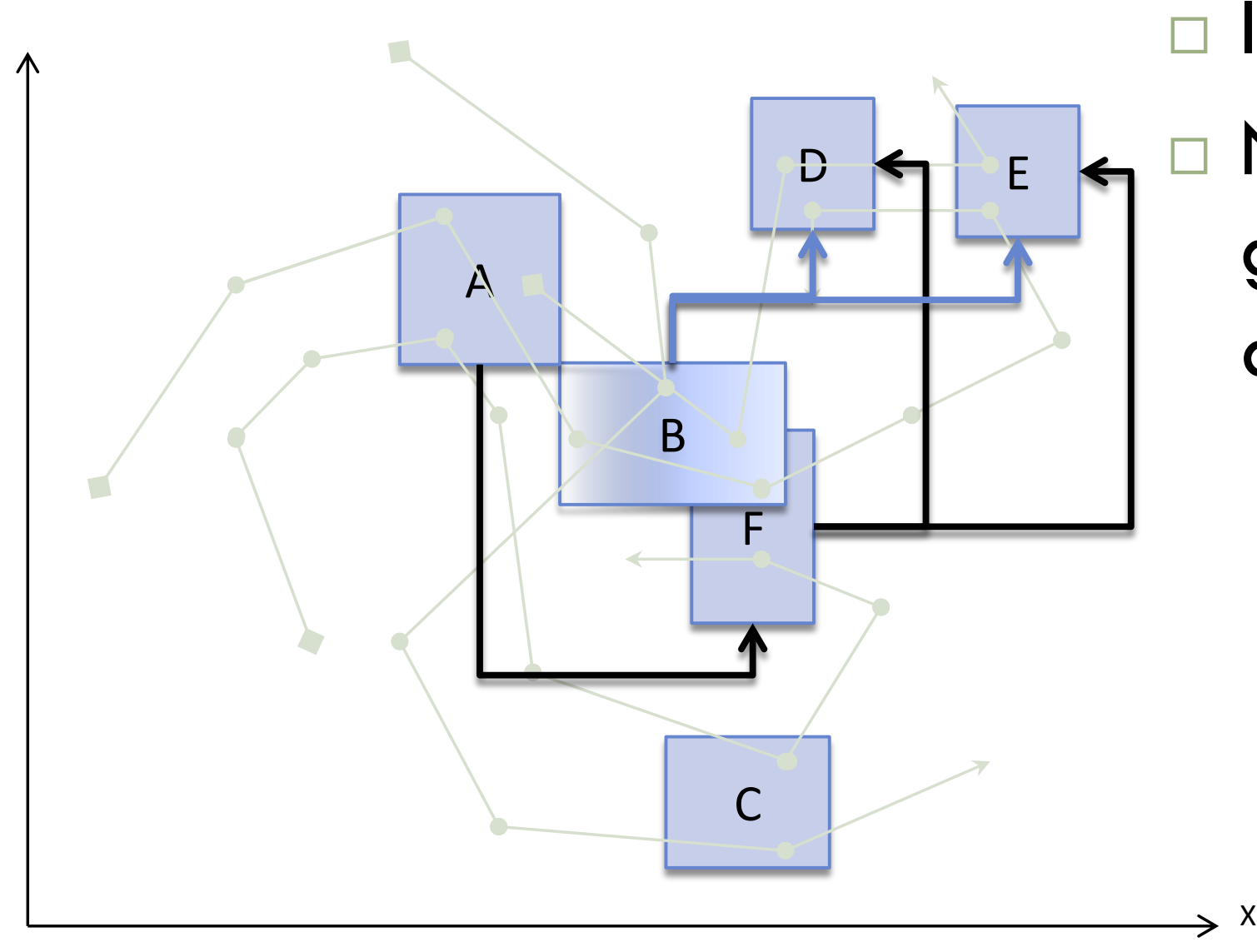

Iteration 4

No more paths to go therefore stops and outputs paths:

$$
\begin{aligned}
& A \rightarrow F \rightarrow D \\
& A \rightarrow F \rightarrow E \\
& B \rightarrow D \\
& B \rightarrow E
\end{aligned}
$$




\section{Results}

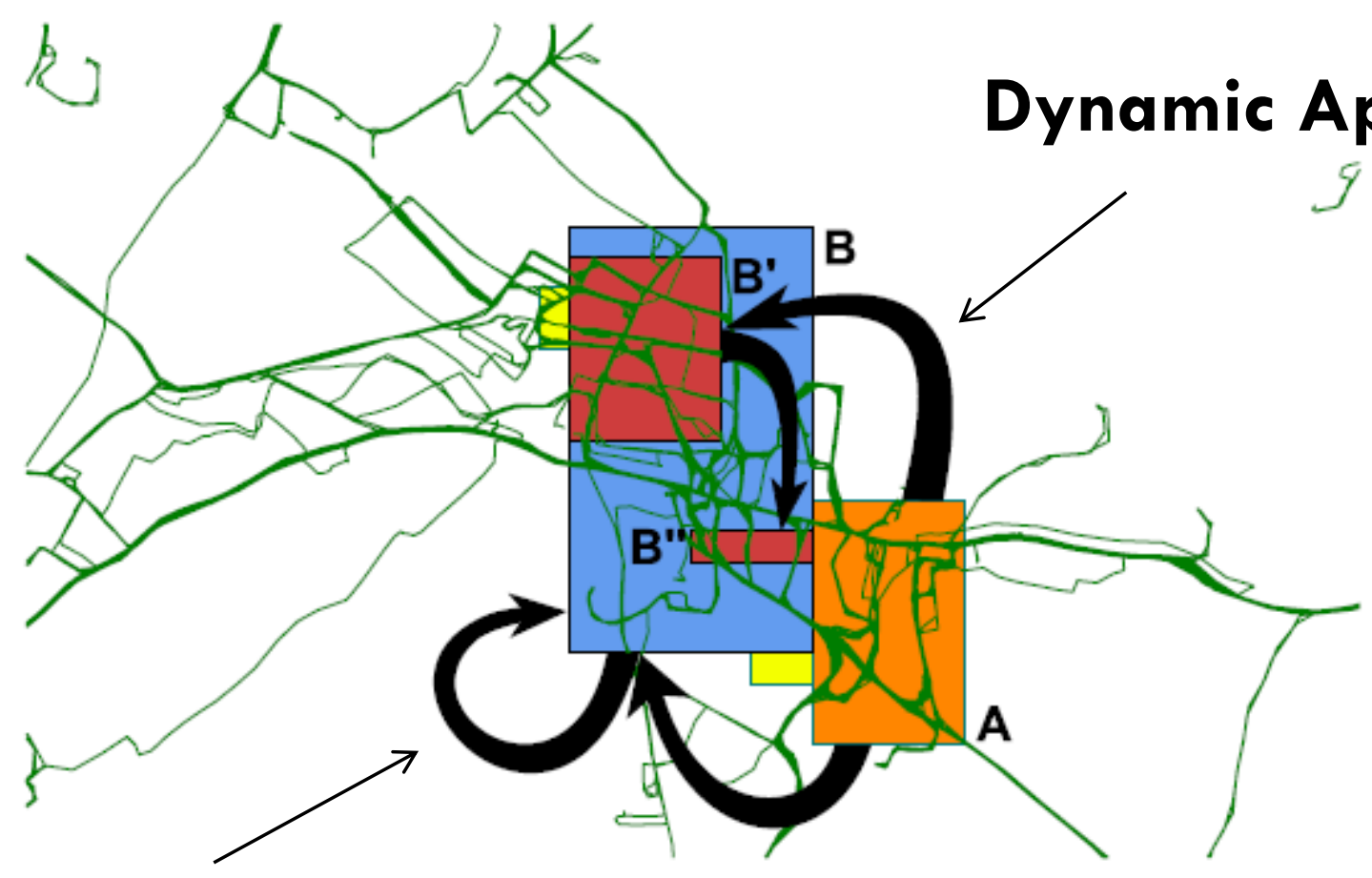

Static Approach

Trajectory Pattern Mining: Fosca Giannotti, Micro Nanni, Dino Pedreschi, Fabio Pinelli 


\section{Results}

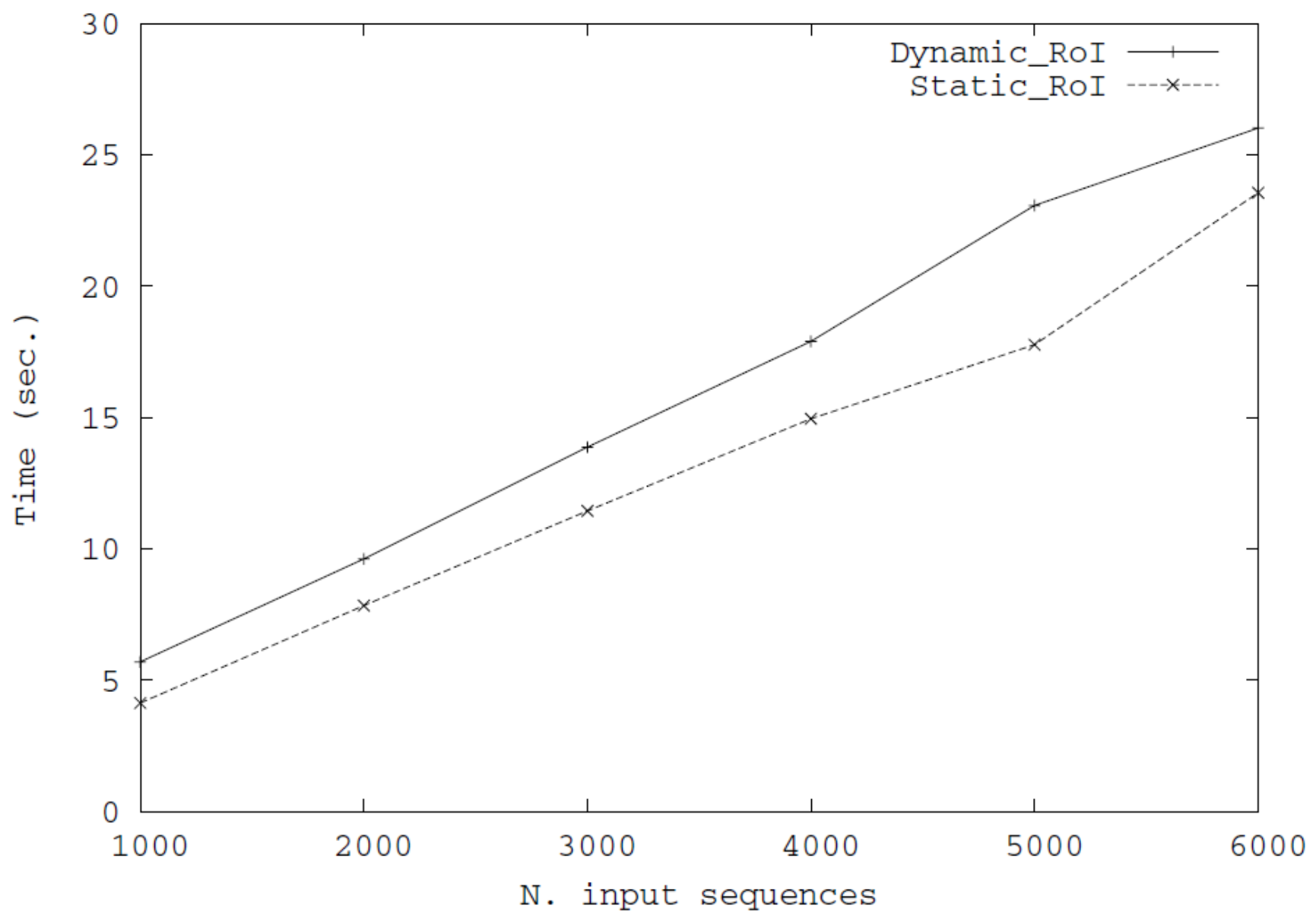

Trajectory Pattern Mining: Fosca Giannotti, Micro Nanni, Dino Pedreschi,

Fabio Pinelli 


\section{Results}

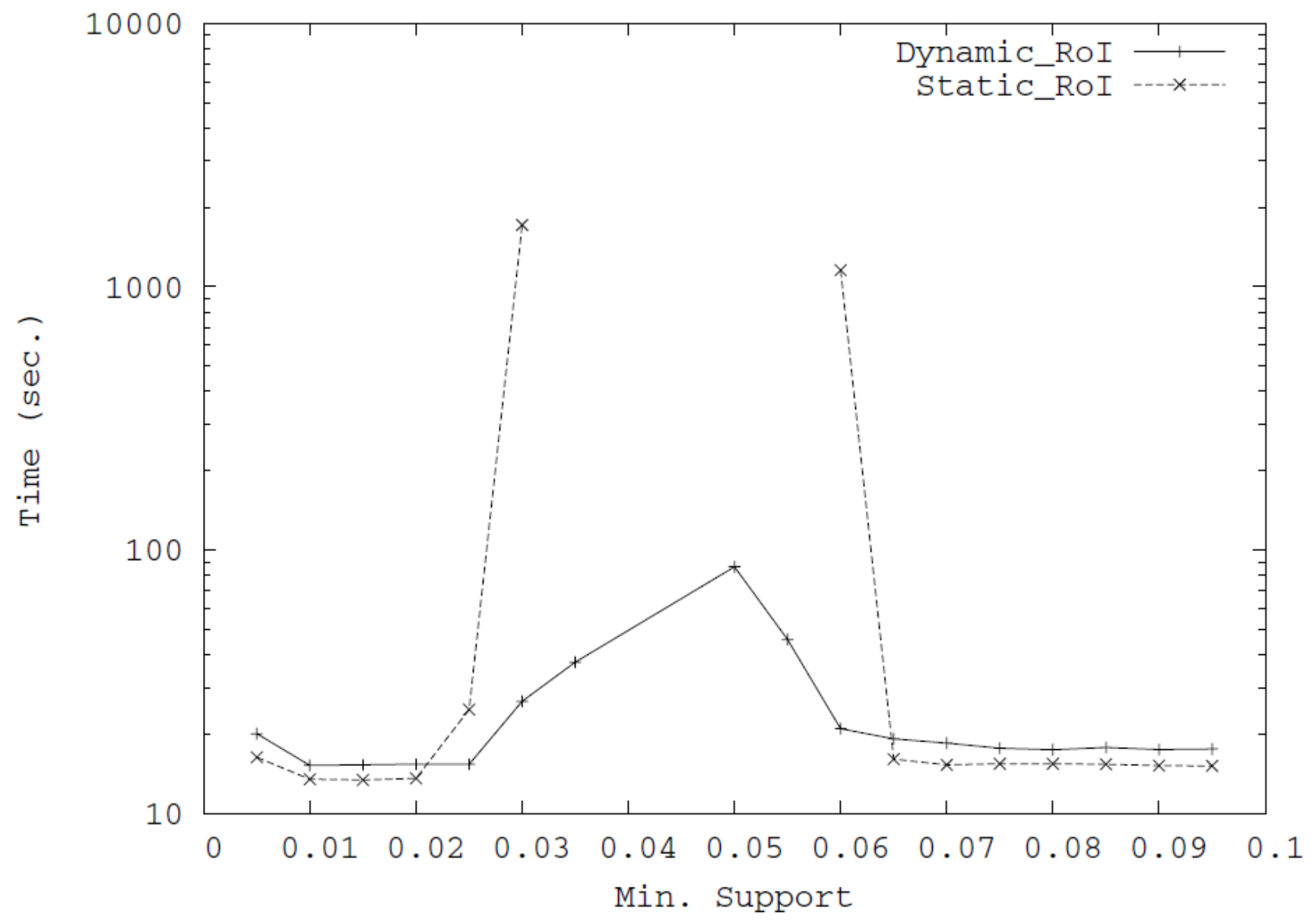

Trajectory Pattern Mining: Fosca Giannotti, Micro Nanni, Dino Pedreschi,

Fabio Pinelli 


\section{To conclude}

$\square$ Lack of comparative results

$\square$ Idea of ROI is good for applications looking for simple and course results.

$\square$ Cited many times for ROI discovery algorithm

$\square$ Where Next: T-Patterns used to predict where a person will move next, given the current sequence.

$\square$ DAEDALUS: Extends SQL to enable TAS queries. 


\section{References}

$\square$ Giannotti, F., Nanni, M., Pinelli, F., and Pedreschi, D. 2007. Trajectory pattern mining. http://doi.acm.org/10.1145/1281192.1281230

$\square$ F. Giannotti, M. Nanni, and D. Pedreschi. Efficient mining of sequences with temporal annotations http://www.siam.org/meetings/sdm06/proceedings/032giannottif. pdf

$\square$ Monreale, A., Pinelli, F., Trasarti, R., and Giannotti, F. 2009. WhereNext: a location predictor on trajectory pattern mining http://doi.acm.org/10.1145/1557019.1557091

$\square$ Ortale, R., Ritacco, E., Pelekis, N., Trasarti, R., Costa, G., Giannotti, F., Manco, G., Renso, C., and Theodoridis, Y. 2008. The DAEDALUS framework: progressive querying and mining of movement data. http://doi.acm.org/10.1145/1463434.1463497 


\section{Thanks!}

Trajectory Pattern Mining: Fosca Giannotti, Micro Nanni, Dino Pedreschi,

Fabio Pinelli 